\title{
Neuropathological interpretation of stimulated Raman histology images of brain and spine tumors: part B
}

\author{
Jakob Straehle ${ }^{1}$ - Daniel Erny ${ }^{2}$. Nicolas Neidert ${ }^{1,3} \cdot$ Dieter Henrik Heiland $^{1,3,4,5,6} \cdot$ Amir El Rahal $^{1}$. \\ Vlad Sacalean $^{1,3}$. David Steybe ${ }^{7} \cdot$ Rainer Schmelzeisen ${ }^{6,7}$. Andreas Vlachos ${ }^{6,8,9,10}$ - Boris Mizaikoff ${ }^{11,12}$. \\ Peter Christoph Reinacher ${ }^{6,13,14} \cdot$ Volker Arnd Coenen $^{6,13} \cdot$ Marco Prinz $^{2,6,9,15} \cdot$ Jürgen Beck ${ }^{1,4,6,9} \cdot$ Oliver Schnell $^{1,6}$ (])
}

Received: 25 October 2021 / Revised: 22 November 2021 / Accepted: 26 November 2021 / Published online: 10 December 2021

(c) The Author(s) 2021

\begin{abstract}
Intraoperative histopathological examinations are routinely performed to provide neurosurgeons with information about the entity of tumor tissue. Here, we quantified the neuropathological interpretability of stimulated Raman histology (SRH) acquired using a Raman laser imaging system in a routine clinical setting without any specialized training or prior experience. Stimulated Raman scattering microscopy was performed on 117 samples of pathological tissue from 73 cases of brain and spine tumor surgeries. A board-certified neuropathologist - novice in the interpretation of SRH - assessed image quality by scoring subjective tumor infiltration and stated a diagnosis based on the SRH images. The diagnostic accuracy was determined by comparison to frozen hematoxylin-eosin (H\&E)-stained sections and the ground truth defined as the definitive neuropathological diagnosis. The overall SRH imaging quality was rated high with the detection of tumor cells classified as inconclusive in only $4.2 \%$ of all images. The accuracy of neuropathological diagnosis based on SRH images was $87.7 \%$ and was non-inferior to the current standard of fast frozen H\&E-stained sections (87.3 vs. 88.9\%, $p=0.783$ ). We found a substantial diagnostic correlation between SRH-based neuropathological diagnosis and H\&E-stained frozen sections $(\kappa=0.8)$. The interpretability of intraoperative SRH imaging was demonstrated to be equivalent to the current standard method of H\&E-stained frozen sections. Further research using this label-free innovative alternative vs. conventional staining is required to determine to which extent SRH-based intraoperative decision-making can be streamlined in order to facilitate the advancement of surgical neurooncology.
\end{abstract}

Keywords Stimulated Raman histology (SRH) $\cdot$ Neuropathology $\cdot$ Diagnostic accuracy $\cdot$ Neurosurgery $\cdot$ NIO $\cdot$ H\&Estained frozen section

\section{Introduction}

State-of-the-art intraoperative histopathological diagnosis in a neurosurgical setting is routinely performed using fast frozen section and H\&E staining. This technique is limited by the amount of samples that can be processed in real-time in a routine clinical setting, as for a single sample there is a delay of 15-30 min from tissue removal to histopathological diagnosis. Therefore, an exhaustive histopathological sampling of tumor margins to assist neurosurgical decision-making in real-time is currently not available during routine tumor resections. Decisions on the extent of resection rather rely on

Oliver Schnell

oliver.schnell@uniklinik-freiburg.de

Extended author information available on the last page of the article subjective criteria such as visual and haptic impressions of the neurosurgeon, 5-aminolevulenic acid (5-ALA)-mediated fluorescence [24], intraoperative MRI [22], ultrasound, neuromonitoring [21], or neuronavigation.

A technological breakthrough $[6,7]$ leading to the development of a mobile stand-alone fiber-laser-based stimulated Raman scattering microscope (NIO Invenio Imaging Inc.) enables to perform stimulated Raman histology (SRH) with minimal delay ( $\sim 3 \mathrm{~min}$ ) label-free (i.e., without additional staining) directly in the operating theater [17].

Compared to conventional H\&E staining, SRH has several advantages such as the ease of use, the time of data processing, and the digital nature of the obtained images. Digitalization allows remote consultations and image processing using automated classifiers and machine learning routines [9]. 
At the core of the novel application of SRH is the fundamental understanding of the obtained SRH images. Despite obvious analogies between SRH images and H\&E-stained sections, there are also differences. Firstly, tissue specimens used in SRH are squash preparations where characteristic cytoarchitectonic features of the tissue may be disrupted. Secondly, SRH images highlight cellular features - mainly tumoral and glia fibers - but also putative axons that are usually not evident on conventional $\mathrm{H} \& \mathrm{E}$ stains. The incorporation of intraoperative SRH imaging into clinical routine requires a close interdisciplinary exchange, whereby neurosurgeons and neuropathologists potentially with the support of expert vibrational spectroscopists become familiar with these new imaging modalities.

Following the introduction of intraoperative SRH imaging at our institution, we have tested the applicability of this technique in a routine clinical setting [16]. The aim of the current study was to (1) quantify the interpretability of SRH images and to (2) evaluate the diagnostic accuracy of SRH compared to the current standard.

\section{Materials and methods}

\section{Tissue acquisition and preparation}

Tissue samples were collected and imaged in accordance with the guidelines of the biobank at the Department of Neurosurgery, Medical Center University of Freiburg, with the approval of the local ethics committee of the University of Freiburg (protocol 5565/15) and with written informed patient consent. One hundred seventeen samples of putative pathological tissue from 73 consecutive surgical cases of brain, spine, and peripheral tumors $(1.6 \pm 0.9$ range from 1 to 5 samples/case) were investigated using stimulated Raman scattering microscopy. Surgeries included tumor resections $(n=67)$ and stereotactic biopsies $(n=6)$. The usual surgical routine was neither disrupted nor influenced.

Samples for SRH images were collected from the adjacent areas of samples extracted for conventional routine diagnostics. SRH was performed as described in detail in the accompanying study [16]. In all cases, pathological tissue was sampled and subject to conventional neuropathological processing (minimal time to diagnosis 2 days). According to our institution's standard of care, in 63 of 73 cases, additional tissue was sampled and processed for intraoperative neuropathological diagnostic using fast frozen H\&E-stained sections.

\section{Sample processing and SRH imaging}

Small (1-3 mm edge size) samples were compressed to a thickness of $230 \mu \mathrm{m}$ on a sample holder designed for use in the Raman imaging system (NIO Slide; NIO Invenio Imaging Inc.). SRH images were generated using a fiberlaser-based stimulated Raman scattering microscope (NIO Laser Imaging System, Invenio Imaging Inc., Santa Clara, CA, USA). Briefly, the raw image data consisted of Raman shifts at wavenumbers $2845 \mathrm{~cm}^{-1}$ and $2940 \mathrm{~cm}^{-1}$, respectively, corresponding to the vibrational frequencies of $\mathrm{C}-\mathrm{H}_{2}$ bonds (primarily characteristic for lipids) and $\mathrm{C}-\mathrm{H}_{3}$ bonds (primarily characteristic for proteins and DNA), respectively. The raw images were generated as sequential line scans of 1000 pixels in width at an imaging depth of $10 \mu \mathrm{m}$ below the coverslip. The nominal pixel size was $467 \mathrm{~nm}$. The images were automatically stitched and converted to SRH (i.e., "virtual H\&E") images via subtraction and the use of a proprietary lookup table (see ref. [17]), which is part of the NIO software package (version 1.5.0). In total, $309 \mathrm{SRH}$ images with an average of $4.2 \pm 2.3$ (range 1 to 12) images per case were generated. In 237 of 309 images, the size of the field of view was nominally set to $2 \times 2 \mathrm{~mm}$ resulting in an effective field of view of 3.06 $\mathrm{mm}^{2}$. The average image size was $3.7 \pm 2.4$ ranging from 0.2 to $19.4 \mathrm{~mm}^{2}$. Images were exported in DICOM format. For ease of use, SRH images were converted to.tif format using custom written scripts (MATLAB R2020a).

\section{Criteria for the neuropathological assessment of SRH images}

The SRH images were presented to a board-certified neuropathologist (D. E.), who had no prior experience in the interpretation of SRH. To evaluate SRH image quality, each image of putative pathological tissue was classified with respect to the assessability of tumor infiltration using a ranked score: (1) infiltration is certain or can be certainly excluded, (2) possible infiltration, and (3) inconclusive, where assessment of infiltration is not possible. Next, the medical information including age, tumor location, and an anonymized brief medical history otherwise identical to the information available at the time of the conventional neuropathological consultation was revealed. Then, based on all SRH images per case, the neuropathologist stated a diagnosis. As a guideline, the following categories were an optional diagnosis: highgrade glioma, low-grade glioma, metastatic brain or spinal tumor, meningioma, CNS lymphoma, pituitary adenoma, sub-/ependymoma, sarcoma, schwannoma, necrosis, reactive gliosis, ganglioglioma, hemangioblastoma, colloid 
cyst, epidermoid, unclear, or other tumors. The written SRH and H\&E-based diagnosis were then classified into the final categories $(n=14)$ by a referee (D.H.H.).

\section{Statistical analysis}

Group comparisons were performed using chi-squared testing. Significance was defined as an alpha level $<5 \%$. Precision and recall between SRH or H\&E-stained frozen section diagnosis and the ground truth (i.e., definite diagnosis) were calculated using the $\mathrm{F}$ measurement function in the FlowSOM package in R (RStudio Version 1.4.1106). $F$ statistic testing was performed using linear models function in $\mathrm{R}$. The inter-rater correlation was calculated using Cohen's kappa coefficient [2] using the psych package in $\mathrm{R}$.

\section{Results}

Seventy-three consecutive neurosurgical cases of brain, spine, and peripheral tumors (Fig. 1a) were investigated using SRH imaging. The mean patient age was $55.9 \pm 18.1$ years with 39 female and 34 male patients. The majority of tumors originated from the brain $(n=63)$ with high-grade gliomas (HGG, $n=17$ ) and brain metastasis $(n=13)$ forming the largest groups (Fig. 1a). In this study, we defined HGG as WHO grade III and IV gliomas, and low-grade gliomas (LGG) as WHO grades I and II. The medical history was classified into 3 groups: (1) novel primary tumors $(n=39),(2)$ recurrent disease $(n=16)$, and (3) primary metastatic disease with known potential origin outside the CNS ( $n=18$ ) (Fig. 1b). While most cases of brain tumors $(37 / 63)$ were primary, the majority of spine tumors $(8 / 10)$ were metastatic with a known primary tumor (Fig. 1b). Examples of SRH images of the 6 largest categories of tumors (cf. Figs. 2j, 3b) are shown in Fig. 1c-h.
Fig. 1 Data and stimulated Raman histology. a Category and location of 73 cranial, spinal, and peripheral (blue) tumors. b Distribution of patient history. $\mathbf{c}-\mathbf{h}$ Illustrative examples of SRH images. c GBM of the left parietal lobe in a 72-year-old female. d 53-yearold male with left frontal oligodendroglioma WHO grade II. e Spinal (TH 2/3) psammomatous meningioma in a 78-year-old male. f Left frontal dural metastasis of an esophageal cancer in a 65-year-old male. g Reactive gliosis with necrotic components (shown) after radiation of a left temporo-occipital melanoma metastasis in a 40-yearold female. h Non-hormone active pituitary adenoma in a 56-year-old male. Scale bars, $100 \mu \mathrm{m}$. Program used to create figure: Adobe Illustrator CS 6

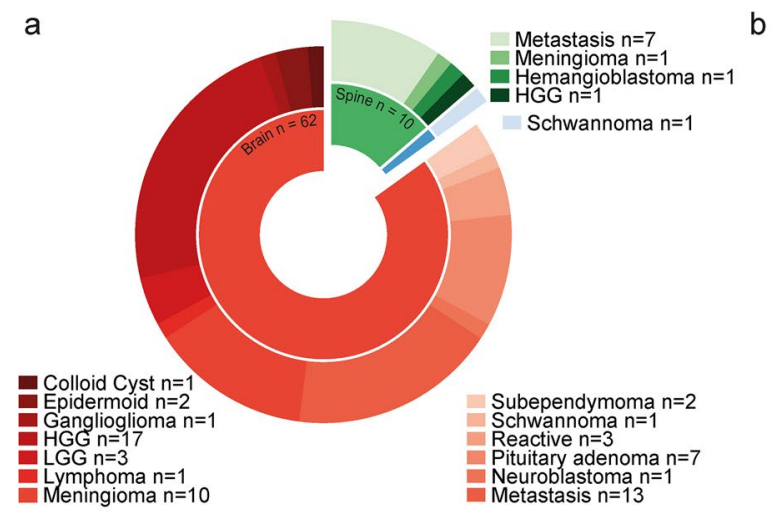

b
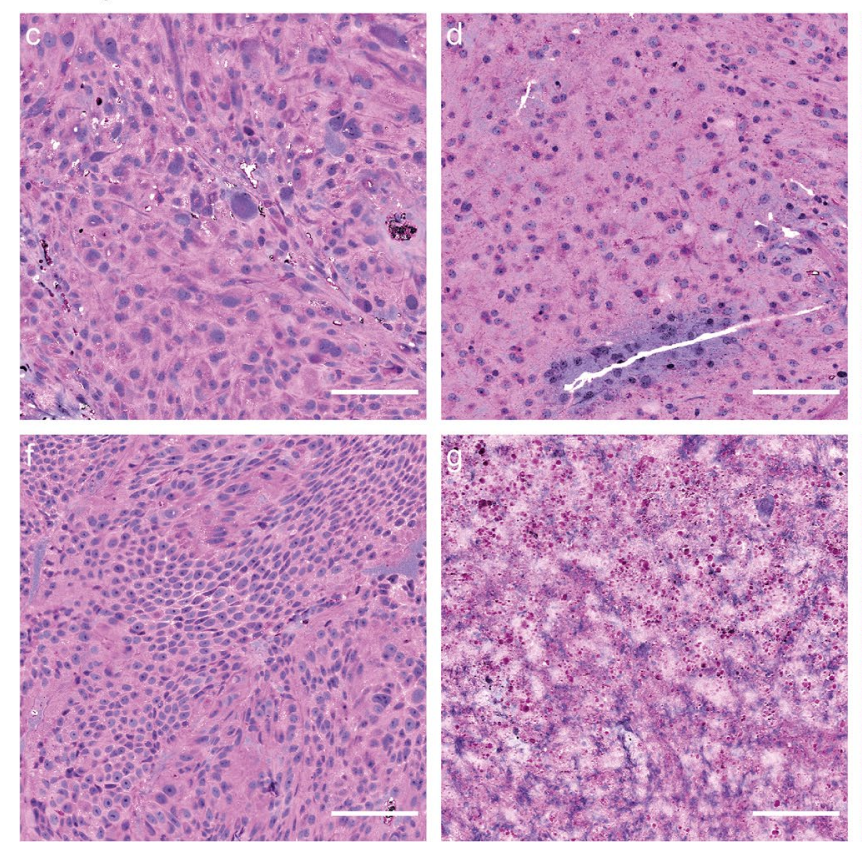

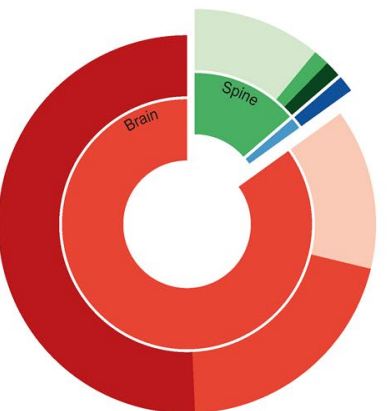

2. Recurrent 3. Metastatic known primary
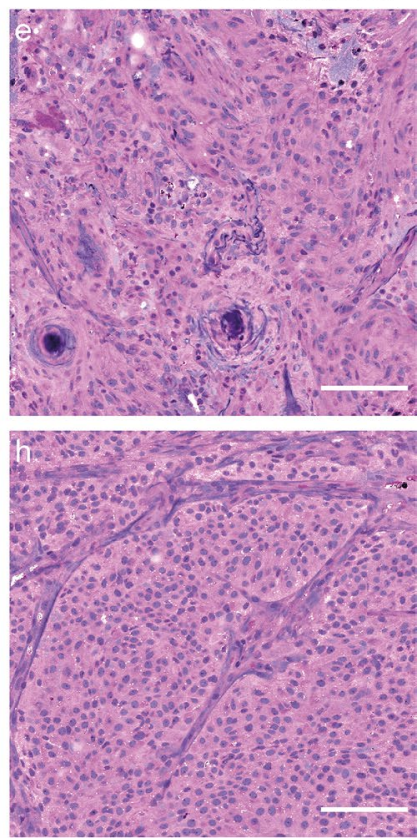

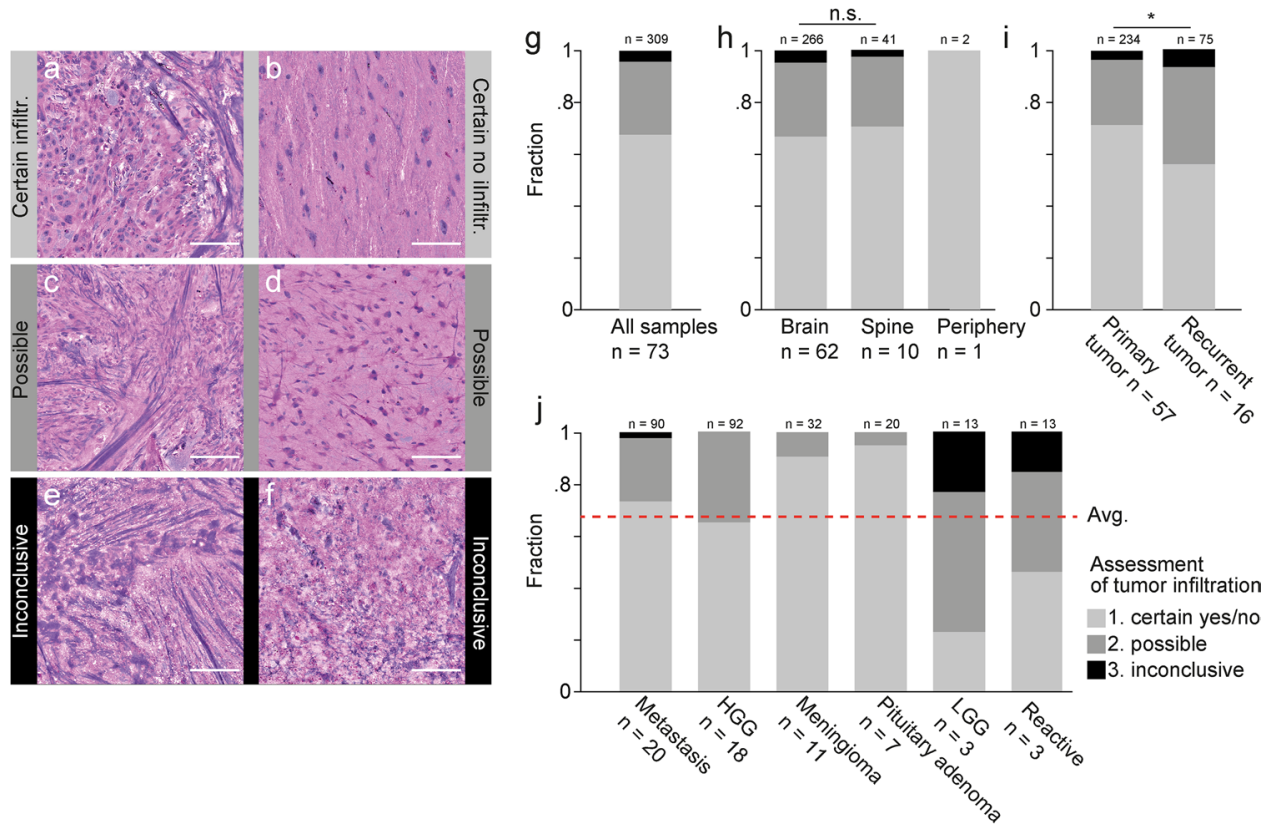

Fig. 2 Assessment of tumor infiltration using SRH imaging. a-f Examples of subjective classification of tumor infiltration in SRH images as certain $(\mathbf{a}, \mathbf{b})$, possible $(\mathbf{c}, \mathbf{d})$, and inconclusive $(\mathbf{e}, \mathbf{f})$. a Certain tumor infiltration in case of a 78-year-old female with metastasis of NSCLC in the right frontal lobe. b Certain absence of tumor infiltration in case of cortical access tissue for resection of a right temporo-occipital GBM. c 72-year-old male patient with spinal metastasis of laryngeal squamous cell carcinoma. d 49-year-old male with recurrent left frontal GBM. e 77-year-old female with recurrent left temporal NSCLC metastasis. f 40-year-old female with

\section{Assessment of tumor infiltration in SRH images}

To evaluate the interpretability of the obtained SRH images $(n=309)$ of putative pathological tissue, we established a ranked score based on the subjective confidence of a neuropathologist, who was novice in the interpretation of SRH images. The presence or absence of tumorous cells was assessed regardless of the underlying pathology. The score comprised 3 classes of tumor infiltration: class 1 certain infiltration (yes/no); class 2 possible infiltration; and class 3 inconclusive (Fig. 2a-f). Overall, $67.6 \%$ of SRH images were classified as class $1,28.2 \%$ as class 2 , and $4.2 \%$ as inconclusive class 3 , respectively (Fig. $2 \mathrm{~g}$ ). The majority of SRH images (10 of 13) classified as class 3 were reported within the first 10 cases that were analyzed. They included necrosis $(n=2)$, fibrous tissue (case of spinal metastasis, $n=1)$, white matter $(n=2)$ (Suppl. Fig. 1), cases of diffuse infiltrating tumor (LGG and neuroblastoma, $n=7$ ), and SRH images taken close to the sample border (sampling error, $n=1)$.

Stratification of subjective interpretability of SRH images by sample origin (Fig. 2h) showed a non-significant metastasis of malignant melanoma in the left temporo-occipital lobe. g Overall assessment of tumor infiltration in 309 SRH images from 73 neurosurgical cases (cf. Fig. 1a). h Stratification of assessment of tumor infiltration according to tumor location and $\mathbf{i}$ the medical history. $\mathbf{j}$ Stratification of assessment of tumor infiltration according to diagnostic category (cf. Fig. 1a). Shown here are the 6 categories that contained $>3$ cases and $>10$ SRH images per category. Red line shows overall average (cf. Fig. 2g). Above all bars are the number of SRH images, below the number of cases per category. Scale bars, $100 \mu \mathrm{m}$. Program used to create figure, Adobe Illustrator CS 6

difference in class 1 confidence in brain vs. spinal tumors ( 66.92 vs. $70.73 \%, p=0.62$ chi-squared). Prior treatment such as radio- and/or chemotherapy decreased the interpretability of SRH images (Fig. 2i). In cases of recurrent disease $(n=16)$ vs. primary tumor cases $(n=57$, group 1 and 3$)$ (Fig. 1b), the class 1 confidence of tumor infiltration was significantly reduced (56.0 vs. $71.4 \%, p=0.013$ chi-squared). Stratification of subjective interpretability of SRH into the six largest tumor categories (cf. Fig. 1c-h) revealed aboveaverage certainty of tumor infiltration in the case of pituitary adenoma, meningioma, and metastasis, while below-average certainty was observed in LGG and reactive gliosis.

\section{Accuracy of neuropathological diagnosis based on SRH images}

The neuropathologist stated a diagnosis based on all available SRH images/case and the medical history. In 2 out of 73 cases (i.e., primary epidermoid in the 4 th ventricle and recurrent GBM in right temporal lobe), a diagnosis was not possible. Using the final neuropathological report as a ground truth, the overall accuracy of the diagnosis based 


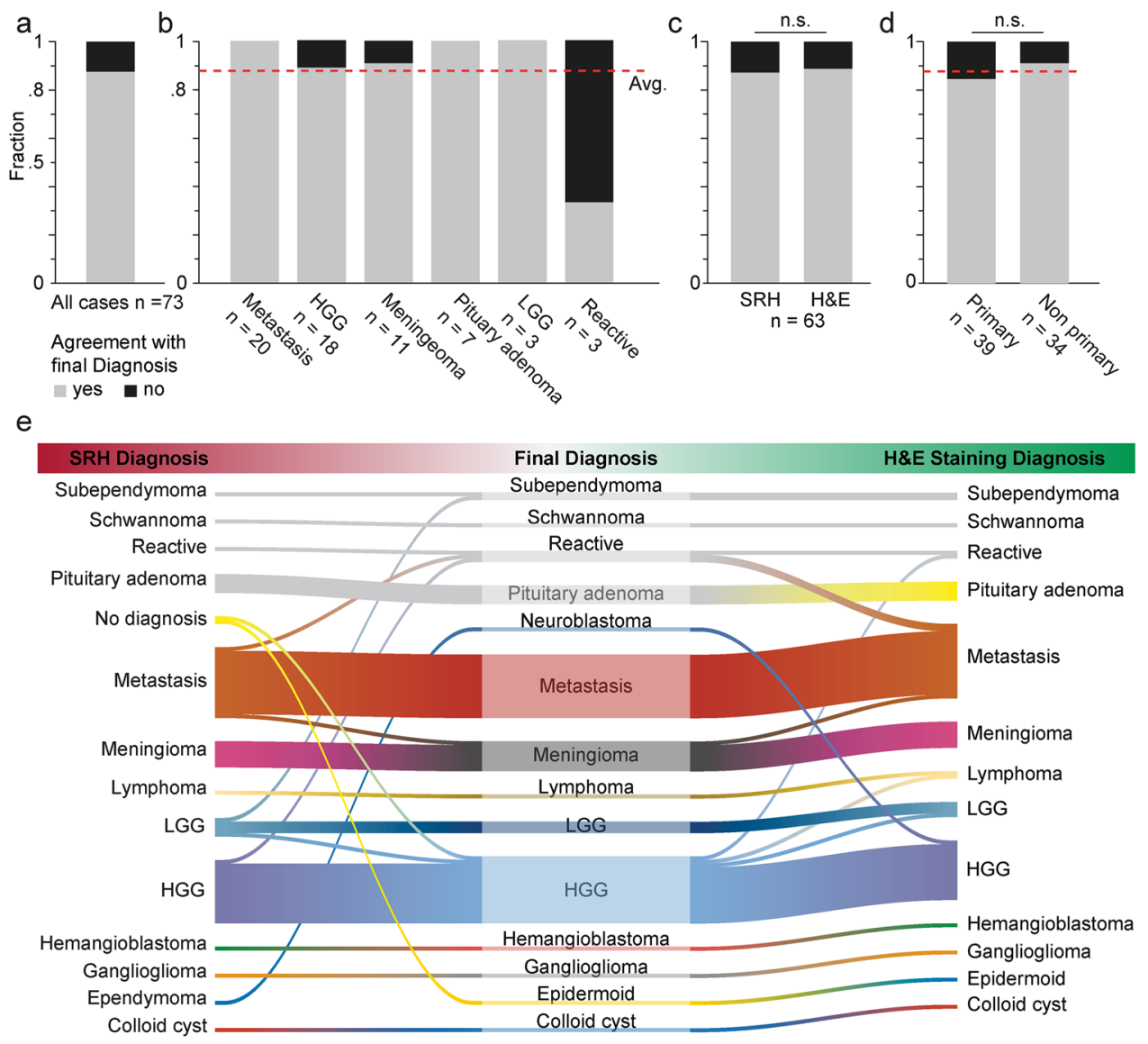

Fig. 3 Accuracy of diagnosis based on medical history and SRH images. A board-certified neuropathologist novice in the assessment of SRH images stated a diagnosis based on the SRH images and the clinical information in 73 cases of cranial, spinal, or peripheral tumors. a Overall agreement of the diagnosis compared to the final neuropathological diagnosis was $87.7 \%$ (cf. red line in $\mathbf{b}$ and $\mathbf{d}$ ). b Stratification of diagnostic accuracy according to tumor entity. Shown here are the 6 entities that contained $>3$ cases. Below all bars are the number of patients per category. $\mathbf{c}$ Non-inferiority of diagnostic accu- racy of SRH vs. conventional fast frozen section using H\&E staining ( 87.3 vs. $88.9 \%, p=0.783$ chi-squared). d Non-significant lower accuracy in primary tumor cases (cf. group 1, Fig. 1b) vs. non-primary cases (cf. groups 2 and 3, Fig. 1b) (84.6 vs. 91.2\%, respectively; $p=0.395$ chi-squared). e River plot showing the correspondence of SRH-based diagnosis (left) and H\&E-stained fast frozen sections (right) to the definitive neuropathological diagnosis (middle) with misclassifications appearing as lane changes. Program used to create figure, Adobe Illustrator CS 6 on SRH imaging was $87.7 \%$ (64 of 73 cases) (Fig. 3a). The diagnostic accuracy based on SRH images was below-average for reactive gliosis, while there was a $100 \%$ accuracy in LGGs, pituitary adenomas, and brain or spine metastasis (Fig. 3b). For HGGs and meningiomas, the diagnostic accuracy using SRH images was $88.9 \%$ and $90.9 \%$, respectively.

\section{Comparison of diagnosis based on SRH images to fast frozen H\&E-stained sections}

A comparison of the SRH-based diagnosis to the current standard in neuropathological diagnosis based on fast frozen $\mathrm{H} \& \mathrm{E}$-stained sections was performed in 63 cases (Fig. 3c, e). The accuracy of diagnosis based on SRH was $87.3 \%$ and $88.9 \%$ based on H\&E-stained sections, respectively. The difference was not significant ( $p=0.783$, chi-squared), demonstrating that the diagnostic accuracy of SRH is en par with H\&E-stained fast frozen sections, which was previously reported [4]. The correlation between the diagnosis based on SRH vs. H\&E-stained sections was determined using Cohen's kappa [2] and was indeed high at $\kappa=0.80$ ([0.70 to 0.91$] 95 \% \mathrm{CI})$. The precision and recall of the diagnosis based on SRH images compared to the ground truth were 0.94 and 0.90 , respectively $(p=2.5 \mathrm{E}-10)$, with a diagnostic of correlation of $\kappa=0.84([0.75,0.94] 95 \% \mathrm{CI}$, unweighted Cohen's kappa). Similarly, the precision and recall for the diagnosis based on H\&E-stained sections were 0.96 and $0.89(p=2.2 \mathrm{E}$ $16)$ with a diagnostic correlation of $\kappa=0.86([0.77,0.96]$ 95\% CI). 


\section{Estimation of bias to diagnostic accuracy}

To estimate bias via the knowledge of the medical history on the neuropathological decision-making, the accuracy of primary tumors (i.e., without any previous neuropathological reports) was compared to the groups of recurrent disease and metastatic tumors with a known primary disease outside the CNS (group 2 and 3) (Fig. 1c). The diagnostic accuracy in the non-primary group was higher, although not reaching statistical significance (84.6 vs. $91.2 \%, p=0.395$, chisquared test) (Fig. 3d).

\section{Discussion}

We report the first experience at our institution with the neuropathological interpretation of ex vivo SRH images, conducted in a routine clinical scenario without any specialized training. Compared to other novel tools for intraoperative histological examination in vivo such as fluorescein-assisted confocal laser endomicroscopy $[8,14]$ and hand-held (multimodal) Raman spectroscopy probes [3, 10], the ex vivo SRH approach described here is limited by the fact that the tissue must be removed. Despite the significant speedup of SRH compared to conventional histopathology [4], the residual delay and the inability to process samples in parallel hinder repeated arbitrary sampling. Rather, a resection strategy including the sequential histopathological examination of multiple locations of interest is required (see below).

\section{Assessment of tumor infiltration in putative pathological samples}

In $67.6 \%$ of all images, we found a high confidence in the assessment of tumor infiltration by a neuropathologist, who was unfamiliar with the interpretation of SRH images. Post hoc analysis revealed that 6 samples contained cortex adjacent to the tumor; therefore, the class of "certain infiltration" also encompassed certain non-infiltrated (i.e., putative healthy) tissue (Fig. 2a, b). We observed a steep learning curve where the majority of images labeled as inconclusive (4.2\%) (Fig. 2g) were reported within the first 10 cases of unsupervised annotation.

Neuropathological diagnosis based on frozen sections of brain tumors in the case of re-operations was shown to have a significantly lower diagnostic accuracy compared to primary brain tumors ( 82 vs. $92 \%$, respectively) [25]. As expected, we found the interpretability of brain tumor infiltration to be significantly reduced in cases of recurrent disease (Fig. 2i), which made up $22 \%$ of all cases in this study (cf. Fig. 1b).

\section{Diagnostic accuracy of SRH-based neuropathological diagnosis}

The accuracy of the SRH-based neuropathological diagnosis from the first samples analyzed at our institution (including the very first) was $87.7 \%$ (Fig. 3a), which was close to the lower bound of the range of diagnostic accuracy (89-98\%) reported for intraoperative frozen section neuropathological diagnosis $[15,25,26]$. It should be noted that the investigators had no prior experience with sample preparation specific to Raman imaging or SRH image interpretation. We expect that the diagnostic accuracy will further increase following ongoing training and the establishment of a standardized protocol.

The SRH-based diagnosis was most accurate for solid primary tumors (e.g., metastasis, meningioma, pituitary adenoma). Misclassifications occurred in cases of recurrent pretreated tumors, reactive gliotic tissue, and HGGs which were also difficult to interpret in conventional H\&E-stained tissues (Fig. 3e). Sampling errors were reported to be another major source of error in the conventional intraoperative neuropathological diagnosis [15]. In this study, spatially similar but not identical samples were processed in parallel for SRH and H\&E. It is possible that distinct parts of the tumor were submitted to either method yet not to the other. Furthermore, only parts of the samples were imaged, which may have led to undersampling of diagnostic features in SRH [17]. This is especially relevant for heterogeneous tumors (e.g., GBM), where necrotic and solid parts are interleaved.

\section{Non-inferiority of SRH diagnosis vs. H\&E-stained frozen section}

As previously reported in a prospective trial by Eichberg et al. where SRH and conventional histology had a similar diagnostic accuracy [4], we found a non-inferiority of the pathological diagnosis based on SRH images compared to conventional fast frozen H\&E-based images (87.3 vs. 88.9\%) (Fig. 3c). The diagnostic correlation between SRH and the ground truth was $\kappa=0.84$, which is similar to the previously reported value of $\kappa=0.83$ [4]. The diagnostic correlation between SRH and H\&E-stained sections reported here $(\kappa=0.8)$ is lower than the near-perfect values reported in Orringer et al. [17] $(\kappa=0.89-0.92)$ but is still considered to be substantial [12]. Recently, Pekmezci et al. [18] showed for resection borders of IDH mutated glioma that the gold standard of immunohistochemistry vs. SRH detected residual tumor at a similar rate ( $56 \%$ vs $49 \%$ ).

We anticipate that neurosurgeons, neuropathologists, and potentially neuroradiologists along with radiotherapists will in future become more familiar with the interpretation of SRH images, which will yield even more accurate interpretations. In addition, it is anticipated that appropriate 

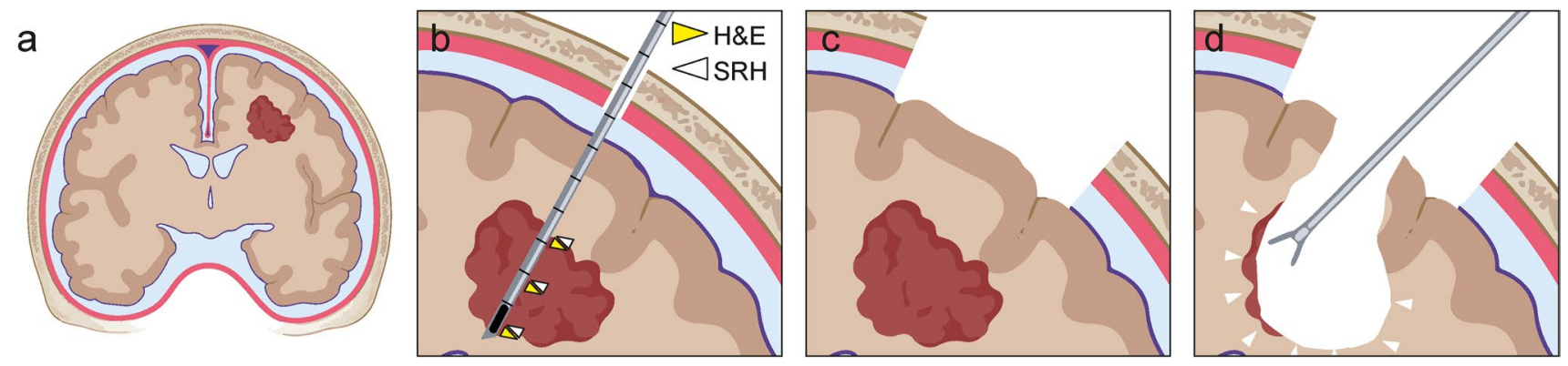

Fig. 4 Workflow for the exploration of histopathological based neurosurgical decision-making. a Coronal schematic of possible neurosurgical approach for brain tumors using intraoperative SRH imaging for detection of the tumor borders. $\mathbf{b}$ Use of a frameless image-guided stereotactic biopsy system through a burr hole at the center of the planned craniotomy. Yellow arrowhead symbolizes samples dedicated

to conventional neuropathological diagnostic, and white arrowhead symbolizes intraoperative SRH. c Craniotomy with potential brain shift and loss of navigation accuracy over time. d Sampling of resection borders to guide resection or for assessment of final resection margins. Program used to create figure, Adobe Illustrator CS 6 and BioRender.com (2021)

deep learning/machine learning strategies [9] will augment the interpretation and classification of the obtained SRH images and further reduce the time to result. The present study provides a quantitative impression of the scenario neurosurgeons and neuropathologists will face when applying advanced imaging technologies in routine clinical settings.

\section{Workflow for the exploration of SRH-based neurosurgical decision-making}

The first positive results reported here together with promising results attesting the diagnostic accuracy of SRH-based imaging similar to the current standard $[4,17,18]$ lead us to conclude - comparable to previous reports $[11,18]$ that intraoperative SRH may be an adequate technique for histopathological based surgical decision-making close to real-time. Modern neuropathological diagnosis relies on a multitude of molecular markers far beyond a mere morphological description $[1,13]$. It is therefore worth noting that intraoperative SRH is not intended to replace conventional pathological techniques but rather work in parallel as a complementary tool in scenarios where a timely feedback is of the essence.

In a possible future surgical scenario of a brain tumor resection (Fig. 4), after exposure of the skull, a frameless stereotactic biopsy [20] of the tumor at several points along a single trajectory via a burr hole centered on the planned craniotomy is performed (duration 10-20 min) (Fig. 4b). The correlated samples are then processed in parallel using SRH and conventional methods using fast frozen sections (duration $\sim 15-30 \mathrm{~min}$ ), validating the SRH images at multiple locations. Performing the biopsy through a burr hole yields the best possible correlation with neuronavigation by minimizing brain shift as well as loss of accuracy with time [23]. Next, craniotomy and durotomy are performed, and the brain is exposed (duration $30 \mathrm{~min}$ ). Ideally, by the time of tumor

resection (Fig. 4c), the conventional fast frozen pathological diagnosis has been reported to the surgeon. Using validated $\mathrm{SRH}$, it will then be possible to analyze the resection borders during the intervention. In case of residual tumor, the surgeon could repeatedly add SRH results to anatomical, fluorescence [5, 24], and neurophysiological [19] criteria to decide continuation or to stop resection.

Supplementary Information The online version contains supplementary material available at https://doi.org/10.1007/s10143-021-01711-1.

Acknowledgements We thank C. Ryba and J. Göldner for technical support.

Author contribution J. S., N. N., J. B., and O. S. designed the study; funding, M. P., D. H. H., R. S., A. V., and J. B.; data acquisition, J. S., N. N., D. S., A. E. R., V. S., and D. E.; P. R. and V. A. C. provided samples and infrastructure; data analysis, J. S., D. H. H., and D. E.; manuscript writing, J. S., J. B., D. H. H., B. M., and O. S. with contributions of all authors.

Funding Open Access funding enabled and organized by Projekt DEAL. J. S. received funding from the Berta-Ottenstein-Programme for Clinician Scientists, Faculty of Medicine, University of Freiburg, Germany. D. E. received funding from the Berta-Ottenstein-Programme for advanced Clinician Scientists, Faculty of Medicine, University of Freiburg, Germany. D. H. H. is funded by the Else KrönerFresenius Foundation. The work is part of the MEPHISTO project (PI, DHH et al.), funded by BMBF (German Ministry of Education and Research) (project number: 031L0260B). A. E. R. received funding from the Nuovo-Soldati Foundation.

Availability of data and material Not applicable.

Code availability Not applicable.

\section{Declarations}

Ethics approval Tissue samples were collected and imaged in accordance with the guidelines of the biobank at the Department of Neurosurgery, Medical Center University of Freiburg, with the approval of the 
local ethics committee of the University of Freiburg (protocol 5565/15) and with written informed patient consent.

Consent to participate Written informed consent from participants is available.

Consent for publication Consent for publication from participants is available.

Conflict of interest The authors declare no competing interests.

Open Access This article is licensed under a Creative Commons Attribution 4.0 International License, which permits use, sharing, adaptation, distribution and reproduction in any medium or format, as long as you give appropriate credit to the original author(s) and the source, provide a link to the Creative Commons licence, and indicate if changes were made. The images or other third party material in this article are included in the article's Creative Commons licence, unless indicated otherwise in a credit line to the material. If material is not included in the article's Creative Commons licence and your intended use is not permitted by statutory regulation or exceeds the permitted use, you will need to obtain permission directly from the copyright holder. To view a copy of this licence, visit http://creativecommons.org/licenses/by/4.0/.

\section{References}

1. Capper D, Jones DTW, Sill M, Hovestadt V, Schrimpf D, Sturm D, Koelsche C, Sahm F, Chavez L, Reuss DE, Kratz A, Wefers AK, Huang K, Pajtler KW, Schweizer L, Stichel D, Olar A, Engel NW, Lindenberg K, Harter PN, Braczynski AK, Plate KH, Dohmen H, Garvalov BK, Coras R, Hölsken A, Hewer E, Bewerunge-Hudler M, Schick M, Fischer R, Beschorner R, Schittenhelm J, Staszewski O, Wani K, Varlet P, Pages M, Temming P, Lohmann D, Selt F, Witt H, Milde T, Witt O, Aronica E, Giangaspero F, Rushing E, Scheurlen W, Geisenberger C, Rodriguez FJ, Becker A, Preusser M, Haberler C, Bjerkvig R, Cryan J, Farrell M, Deckert M, Hench J, Frank S, Serrano J, Kannan K, Tsirigos A, Brück W, Hofer S, Brehmer S, Seiz-Rosenhagen M, Hänggi D, Hans V, Rozsnoki S, Hansford JR, Kohlhof P, Kristensen BW, Lechner M, Lopes B, Mawrin C, Ketter R, Kulozik A, Khatib Z, Heppner F, Koch A, Jouvet A, Keohane C, Mühleisen H, Mueller W, Pohl U, Prinz M, Benner A, Zapatka M, Gottardo NG, Driever PH, Kramm CM, Müller HL, Rutkowski S, von Hoff K, Frühwald MC, Gnekow A, Fleischhack G, Tippelt S, Calaminus G, Monoranu C-M, Perry A, Jones C, Jacques TS, Radlwimmer B, Gessi M, Pietsch T, Schramm J, Schackert G, Westphal M, Reifenberger G, Wesseling P, Weller M, Collins VP, Blümcke I, Bendszus M, Debus J, Huang A, Jabado N, Northcott PA, Paulus W, Gajjar A, Robinson GW, Taylor MD, Jaunmuktane Z, Ryzhova M, Platten M, Unterberg A, Wick W, Karajannis MA, Mittelbronn M, Acker T, Hartmann C, Aldape K, Schüller U, Buslei R, Lichter P, Kool M, Herold-Mende C, Ellison DW, Hasselblatt M, Snuderl M, Brandner S, Korshunov A, von Deimling A, Pfister SM (2018) DNA methylationbased classification of central nervous system tumours. Nature 555:469-474. https://doi.org/10.1038/nature26000

2. Cohen J (1960) A coefficient of agreement for nominal scales. Educ Psychol Measur 20:37-46

3. Desroches J, Jermyn M, Mok K, Lemieux-Leduc C, Mercier J, St-Arnaud K, Urmey K, Guiot M-C, Marple E, Petrecca K (2015) Characterization of a Raman spectroscopy probe system for intraoperative brain tissue classification. Biomed Opt Express $6: 2380-2397$
4. Eichberg DG, Shah AH, Di L, Semonche AM, Jimsheleishvili G, Luther EM, Sarkiss CA, Levi AD, Gultekin SH, Komotar RJ (2019) Stimulated Raman histology for rapid and accurate intraoperative diagnosis of CNS tumors: prospective blinded study. J Neurosurg 134:137-143

5. Fountain DM, Bryant A, Barone DG, Waqar M, Hart MG, Bulbeck H, Kernohan A, Watts C, Jenkinson MD (2021) Intraoperative imaging technology to maximise extent of resection for glioma: a network meta-analysis. Cochrane Database Syst Rev. https://doi.org/10.1002/14651858.CD013630.pub2

6. Freudiger CW, Min W, Saar BG, Lu S, Holtom GR, He C, Tsai JC, Kang JX, Xie XS (2008) Label-free biomedical imaging with high sensitivity by stimulated Raman scattering microscopy. Science 322:1857. https://doi.org/10.1126/science.1165758

7. Freudiger CW, Yang W, Holtom GR, Peyghambarian N, Xie XS, Kieu KQ (2014) Stimulated Raman scattering microscopy with a robust fibre laser source. Nat Photonics 8:153-159. https://doi. org/10.1038/nphoton.2013.360

8. Höhne J, Schebesch K-M, Zoubaa S, Proescholdt M, Riemenschneider MJ, Schmidt NO (2021) Intraoperative imaging of brain tumors with fluorescein: confocal laser endomicroscopy in neurosurgery. Clinical and user experience. Neurosurg Focus FOC 50:E19. https://doi.org/10.3171/2020.11.FOCUS20783

9. Hollon TC, Pandian B, Adapa AR, Urias E, Save AV, Khalsa SSS, Eichberg DG, D’Amico RS, Farooq ZU, Lewis S, Petridis PD, Marie T, Shah AH, Garton HJL, Maher CO, Heth JA, McKean EL, Sullivan SE, Hervey-Jumper SL, Patil PG, Thompson BG, Sagher O, McKhann GM, Komotar RJ, Ivan ME, Snuderl M, Otten ML, Johnson TD, Sisti MB, Bruce JN, Muraszko KM, Trautman J, Freudiger CW, Canoll P, Lee H, Camelo-Piragua S, Orringer DA (2020) Near real-time intraoperative brain tumor diagnosis using stimulated Raman histology and deep neural networks. Nat Med 26:52-58. https://doi.org/10.1038/s41591-019-0715-9

10. Jermyn M, Mercier J, Aubertin K, Desroches J, Urmey K, Karamchandiani J, Marple E, Guiot M-C, Leblond F, Petrecca K (2017) Highly accurate detection of cancer in situ with intraoperative, label-free, multimodal optical spectroscopy. Can Res 77:3942-3950

11. Ji M, Lewis S, Camelo-Piragua S, Ramkissoon SH, Snuderl M, Venneti S, Fisher-Hubbard A, Garrard M, Fu D, Wang AC (2015) Detection of human brain tumor infiltration with quantitative stimulated Raman scattering microscopy. Sci Transl Med 7:309ra163

12. Landis JR, Koch GG (1977) The measurement of observer agreement for categorical data. Biometrics 33:159-174

13. Louis DN, Wesseling P, Aldape K, Brat DJ, Capper D, Cree IA, Eberhart C, Figarella-Branger D, Fouladi M, Fuller GN, Giannini C, Haberler C, Hawkins C, Komori T, Kros JM, Ng H, Orr BA, Park S, Paulus W, Perry A, Pietsch T, Reifenberger G, Rosenblum M, Rous B, Sahm F, Sarkar C, Solomon DA, Tabori U, Bent MJ, Deimling A, Weller M, White VA, Ellison DW (2020) cIMPACTNOW update 6: new entity and diagnostic principle recommendations of the cIMPACT-Utrecht meeting on future CNS tumor classification and grading. Brain Pathol 30:844-856. https://doi. org/10.1111/bpa.12832

14. Martirosyan NL, Eschbacher JM, Kalani MYS, Turner JD, Belykh E, Spetzler RF, Nakaji P, Preul MC (2016) Prospective evaluation of the utility of intraoperative confocal laser endomicroscopy in patients with brain neoplasms using fluorescein sodium: experience with 74 cases. Neurosurg Focus FOC 40:E11. https://doi.org/ 10.3171/2016.1.FOCUS15559

15. Meyer M, Keith-Rokosh J, Reddy H, Megyesi J, Hammond RR (2010) Sources of error in neuropathology intraoperative diagnosis. Can J Neurol Sci 37:620-624. https://doi.org/10.1017/S0317 167100010799

16. Neidert N, Straehle J, Erny D, Sacalean V, El Rahal A, Steybe D, Schmelzeisen R, Vlachos A, Reinacher PC, Coenen VA, Mizaikoff 
B, Heiland DH, Prinz M, Beck J, Schnell O (2021) Stimulated Raman histology in the neurosurgical workflow of a major European neurosurgical center - part A (in press)

17. Orringer DA, Pandian B, Niknafs YS, Hollon TC, Boyle J, Lewis S, Garrard M, Hervey-Jumper SL, Garton HJL, Maher CO, Heth JA, Sagher O, Wilkinson DA, Snuderl M, Venneti S, Ramkissoon SH, McFadden KA, Fisher-Hubbard A, Lieberman AP, Johnson TD, Xie XS, Trautman JK, Freudiger CW, Camelo-Piragua S (2017) Rapid intraoperative histology of unprocessed surgical specimens via fibre-laser-based stimulated Raman scattering microscopy. Nat Biomed Eng 1:0027. https://doi.org/10.1038/ s41551-016-0027

18. Pekmezci M, Morshed RA, Chunduru P, Pandian B, Young J, Villanueva-Meyer JE, Tihan T, Sloan EA, Aghi MK, Molinaro AM, Berger MS, Hervey-Jumper SL (2021) Detection of glioma infiltration at the tumor margin using quantitative stimulated Raman scattering histology. Sci Rep 11:12162. https://doi.org/ 10.1038/s41598-021-91648-8

19. Raabe A, Beck J, Schucht P, Seidel K (2014) Continuous dynamic mapping of the corticospinal tract during surgery of motor eloquent brain tumors: evaluation of a new method. J Neurosurg 120:1015-1024

20. Ringel F, Ingerl D, Ott S, Meyer B (2009) VarioGuide: a new frameless image-guided stereotactic system — accuracy study and clinical assessment. Oper Neurosurg 64:ons365-ons373. https:// doi.org/10.1227/01.NEU.0000341532.15867.1C
21. Seidel K, Schucht P, Beck J, Raabe A (2020) Continuous dynamic mapping to identify the corticospinal tract in motor eloquent brain tumors: an update. J Neurol Surg A Cent Eur Neurosurg 81:105-110

22. Senft C, Bink A, Franz K, Vatter H, Gasser T, Seifert V (2011) Intraoperative MRI guidance and extent of resection in glioma surgery: a randomised, controlled trial. Lancet Oncol 12:997-1003

23. Stieglitz LH, Fichtner J, Andres R, Schucht P, Krähenbühl A-K, Raabe A, Beck J (2013) The silent loss of neuronavigation accuracy: a systematic retrospective analysis of factors influencing the mismatch of frameless stereotactic systems in cranial neurosurgery. Neurosurgery 72:796-807

24. Stummer W, Pichlmeier U, Meinel T, Wiestler OD, Zanella F, Reulen H-J (2006) Fluorescence-guided surgery with 5-aminolevulinic acid for resection of malignant glioma: a randomised controlled multicentre phase III trial. Lancet Oncol 7:392-401. https://doi.org/10. 1016/S1470-2045(06)70665-9

25. Tofte K, Berger C, Torp SH, Solheim O (2014) The diagnostic properties of frozen sections in suspected intracranial tumors: a study of 578 consecutive cases. Surg Neurol Int 5(1):170

26. Uematsu Y, Owai Y, Okita R, Tanaka Y, Itakura T (2007) The usefulness and problem of intraoperative rapid diagnosis in surgical neuropathology. Brain Tumor Pathol 24:47-52

Publisher's note Springer Nature remains neutral with regard to jurisdictional claims in published maps and institutional affiliations.

\section{Authors and Affiliations}

\section{Jakob Straehle ${ }^{1}$ - Daniel Erny ${ }^{2} \cdot$ Nicolas Neidert $^{1,3} \cdot$ Dieter Henrik Heiland $^{1,3,4,5,6} \cdot$ Amir El Rahal $^{1}$. Vlad Sacalean ${ }^{1,3}$. David Steybe ${ }^{7}$. Rainer Schmelzeisen ${ }^{6,7}$. Andreas Vlachos ${ }^{6,8,9,10}$ - Boris Mizaikoff ${ }^{11,12}$. Peter Christoph Reinacher ${ }^{6,13,14} \cdot$ Volker Arnd Coenen $^{6,13} \cdot$ Marco Prinz $^{2,6,9,15} \cdot$ Jürgen Beck ${ }^{1,4,6,9} \cdot$ Oliver Schnell $^{1,6}(\mathbb{C}$}

1 Department of Neurosurgery, Medical Center, University of Freiburg, Freiburg, Germany

2 Institute of Neuropathology, Faculty of Medicine, University of Freiburg, Freiburg, Germany

3 Microenvironment and Immunology Research Laboratory, Medical Center, University of Freiburg, Freiburg, Germany

4 Comprehensive Cancer Center Freiburg (CCCF), Faculty of Medicine and Medical Center, University of Freiburg, Freiburg, Germany

5 German Cancer Consortium (DKTK), partner site Freiburg, Freiburg, Germany

6 Medical Faculty of Freiburg University, Freiburg, Germany

7 Department of Oral and Maxillofacial Surgery, Medical Center, University of Freiburg, Freiburg, Germany

8 Department of Neuroanatomy, Institute of Anatomy and Cell Biology, Faculty of Medicine, University of Freiburg, Freiburg, Germany
9 Center for Basics in NeuroModulation (NeuroModulBasics), Faculty of Medicine, University of Freiburg, Freiburg, Germany

10 Center Brain Links Brain Tools, University of Freiburg, Freiburg, Germany

11 Institute of Analytical and Bioanalytical Chemistry, Ulm University, Ulm, Germany

12 Hahn-Schickard Institute for Microanalysis Systems, Ulm, Germany

13 Department of Stereotactic and Functional Neurosurgery, Medical Center, University of Freiburg, Freiburg, Germany

14 Fraunhofer Institute for Laser Technology (ILT), Aachen, Germany

15 Signalling Research Centres BIOSS and CIBSS, University of Freiburg, Freiburg, Germany 